\title{
First look at CKM parameters from early Belle II data
}

\section{Isabelle Ripp-Baudot ${ }^{* \dagger}$}

IPHC Strasbourg, CNRS/IN2P3, Université de Strasbourg, France.

E-mail: rippein2p3.fr

After its commissioning in 2018, the Belle II experiment has started registering first physics data delivered by SuperKEKB in 2019. The Belle II physics program focuses on the search for physics beyond the standard model with precise measurements in the flavour sector. The huge targeted dataset and the $B$-factory particular collision scheme will help to significantly improve the experimental precision on the three angles of the $b d$ CKM unitarity triangle. These measurements are based on time-dependent $C P$ asymmetry analyses for the angles $\phi_{1}$ and $\phi_{2}$, and on the reconstruction of many $B$ and $D$ hadronic decay modes for $\phi_{3}$. In this proceeding article are reported the first look at these ingredients reconstructed with early Belle II data, and the expected Belle II sensitivity on $\phi_{1}, \phi_{2}$ and $\phi_{3}$ with more data.

The European Physical Society Conference on High Energy Physics - EPS-HEP2019 10-17 July 2019

Ghent, Belgium

\footnotetext{
${ }^{*}$ Speaker.

${ }^{\dagger}$ on behalf of the Belle II collaboration
} 


\section{Introduction}

The previous generation of $B$-factories, BaBar at PEP-II and Belle at KEKB, observed $C P$ violation in the $B$ meson system for the first time and assessed the Cabibbo-Kobayashi-Maskawa (a.k.a. CKM) formalism, with about $1 \mathrm{ab}^{-1}$ of data in the end. Their successor Belle II at SuperKEKB aims at building on their great success and on the excellent accumulated skills, and focuses on the search for new physics beyond the standard model of particle physics, with measurements of unprecedented precision in the heavy quark and charged lepton sectors. A siginificant part of the Belle II physics program is devoted to Flavour Changing Neutral Current process studies to try to untangle the new physics. Such indirect searches of quantum manifestation of new particles are potentially sensitive to a higher mass scale of the unknown new physics than with the direct searches pursued at high energy at the LHC. Moreover the particular collision scheme of $B$-factories allows interesting complementarity with $\mathrm{LHCb}$ based on unique capabilities as far as inclusive measurements are concerned, as well as analyses implying missing energy and neutrals like $\gamma, K_{S}^{0}, K_{L}^{0}, \pi^{0}$ and $\eta$.

SuperKEKB is an asymmetric $e^{+} e^{-}$collider with center-of-mass energies around $\Upsilon$ invariant masses, with the main focus on the $\Upsilon(4 \mathrm{~S})$ decaying to pairs of neutral or charged $B$ mesons. It is based on a new nano-beam collision scheme, with relatively high beam currents and a large beam crossing angle, aiming at reaching around 2025 an instantaneous luminosity 40 times higher than ever achieved, of $8 \times 10^{35} \mathrm{~cm}^{-2} \mathrm{~s}^{-1}$. With respect to BaBar and Belle, this collision scheme implies however a reduced center-of-mass boost, impacting mainly time dependent measurements. It induces unfortunately also a dramatic increase of what is called the machine background, that must be kept under control to achieve the ambitious Belle II physics program.

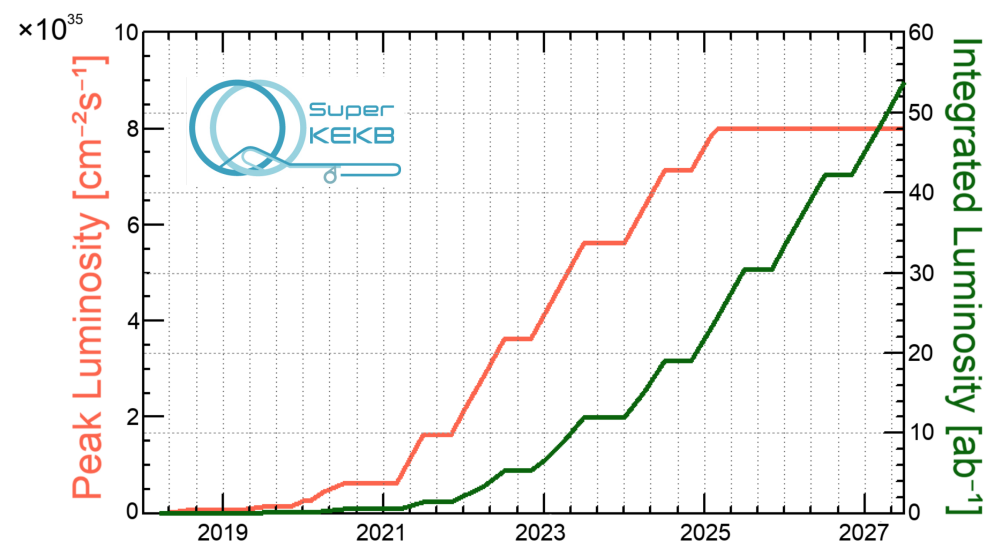

Figure 1: SuperKEKB planned instantaneous (red) and integrated (green) luminosity increases with time.

Figure 1 shows the calendar of SuperKEKB runs, with instantaneous and integrated luminosity plans. After a smooth start in 2019, the Belle and BaBar datasets should be overpassed by end of 2021 and the design luminosity reached in 2025 , to accumulate $50 \mathrm{ab}^{-1}$ by 2027 . The Belle II experiment (excepting the completed inner tracker system) was commissioned in spring 2018, with the main purpose of registering the first collisions of nano-beams and studying the machine background impact in the Belle II detector. The Belle II physics run started in 2019 and the ex- 
periment was switched off end of June for the summer shutdown, a few days before this report. Belle II accumulated in total $6.5 \mathrm{fb}^{-1}$ at $\Upsilon(4 \mathrm{~S})$ in addition to $0.8 \mathrm{fb}^{-1}$ of off peak collisions with energy $60 \mathrm{MeV}$ lower. SuperKEKB instantaneous luminosity exceeded $10^{34} \mathrm{~cm}^{-2} \mathrm{~s}^{-1}$ during this run. Belle II was switched off at the highest reached luminosity due to too high beam induced backround, dominated by beam-gas interaction due to vacuum conditions in the new Low Energy Ring, that are expected to largely improve in the future.

In this document we present a glimpse of first Belle II results with early data registered in 2019 , related to the measurement of the three angles $\phi_{1}, \phi_{2}$ and $\phi_{3}$ of the $b d$ CKM unitarity triangle. These measurements are based on time-dependent $C P$ asymmetry analyses for the angles $\phi_{1}$ and $\phi_{2}$, and on the reconstruction of many $B$ and $D$ hadronic decay modes for $\phi_{3}$. Expected improved experimental precision on the three angles, made possible thanks to the huge Belle II targeted dataset and to the $B$-factory particular collision scheme, is also discussed.

\section{Ingredients of time-dependent $C P$ asymmetry measurements}

The two angles $\phi_{1}$ and and $\phi_{2}$ can be determined by measuring time-dependent $C P$ asymmetries of neutral $B$ meson decays. The time dependence can be expressed as:

$$
\mathscr{P}(\Delta t, q)=\frac{e^{\frac{-|\Delta t|}{\tau_{B^{0}}}}}{4 \tau_{B^{0}}}\left[1+q\left(\mathscr{A}_{C P} \cos \Delta m_{d} \Delta t+\mathscr{S}_{C P} \sin \Delta m_{d} \Delta t\right)\right]
$$

where $\tau_{B^{0}}$ is the lifetime of the $B^{0}$ meson and $\Delta m_{d}$ is the mass difference between the two $B^{0}$ physics eigenstates. The two $C P$ parameters $\mathscr{A}_{C P}$ and $\mathscr{S}_{C P}$ are related respectively to direct and indirect $C P$ violation. As long as penguin contributions can be neglected in the process considered to determine $\phi_{1}$ or $\phi_{2}, \mathscr{A}_{C P}=0$ and $\mathscr{S}_{C P}=\sin 2 \phi_{1,2}$. Two key ingredients are needed to measure time dependent $C P$-asymmetries:

- The flavour $q$ of the $B$ meson decaying to the $C P$ eigenstate. This flavour is inferred unambiguously from the flavour of the companion meson called $B_{T A G}$, thanks to the fact that the pair of $B$ mesons is produced in a quantum entangled state at $\Upsilon(4 S)$ center-of-mass. This powerful trick is a monopoly of $B$-factories.

- The time interval $\Delta t$ between the moment when the flavour of the signal $B$ meson is known and the moment when it decays into the $C P$ eigenstate. It is actually estimated based on the flight distance $\Delta z$ along the boost axis and between the $2 B$ mesons.

The Belle II flavour tagger is a sophisticated algorithm that combines many multivariate classifiers into one. Its performance to identify the flavour $q$ of the signal $B$ meson is expressed as an effective tagging efficiency $\sum \varepsilon_{i} \times\left(1-2 \omega_{i}\right)^{2}$, depending on the efficiency $\varepsilon_{i}$ to identify the flavour of the $B$ meson with the $i^{\text {th }}$ classifier, but also on a dilution factor due to flavour mistag $\omega_{i}$, that decreases the observed $C P$ asymmetry. In Belle II the performance of the flavour tagger is expected to be significantly improved with respect to Babar and Belle. Effective efficiency measured with Belle II simulated data is $37 \%$ [1], thanks to more tagger categories and more input variables, in addition to better detector performances in particular on particle identification. Further improvements are moreover expected, in particular with on-going developments based on Deep Neural Networks. 
At SuperKEKB the beam asymmetry was reduced by $2 / 3$ with respect to KEKB. Consequently it was decided to reduce the radius of the beam pipe to $1 \mathrm{~cm}$ in the interaction region and an innermost pixelated tracking detector was installed end of 2018 in Belle II, at the closest distance possible to the interaction point at a radius of $1.4 \mathrm{~cm}$. In terms of track impact parameter resolution, almost a factor of 2 improvement is expected over the whole momentum range, resulting in an improved resolution on the flight distance $\Delta z$ and helping maintaining at least the same accuracy on $\Delta t$ as achieved in Belle, despite the reduced flight distance in the detector. Furthermore the vertex detector coverage was extended, resulting in a $30 \%$ better acceptance, important in particular for $K_{S}^{0}$ reconstruction. And finally also the nanobeam-spot size, reduced to $(6 \times 0.06 \times 150) \mu \mathrm{m}^{3}$ to be compared with $(120 \times 5 \times 8000) \mu \mathrm{m}^{3}$ previously in Belle, will improve the accuracy on the $B$ reconstructed decay vertex. However beam-constrained vertexing methods commonly used in $\mathrm{BaBar}$ and Belle must be refined to build an unbiased estimator of the $B$ decay point in Belle II. Finally the resolution achieved on $\Delta t$ is expected to be better than $0.8 \mathrm{ps}$ (see figure 2) and largely dominated by the resolution on the tag-side vertex, which is inclusively reconstructed with the rest of the event once the signal $B$ meson is reconstructed.

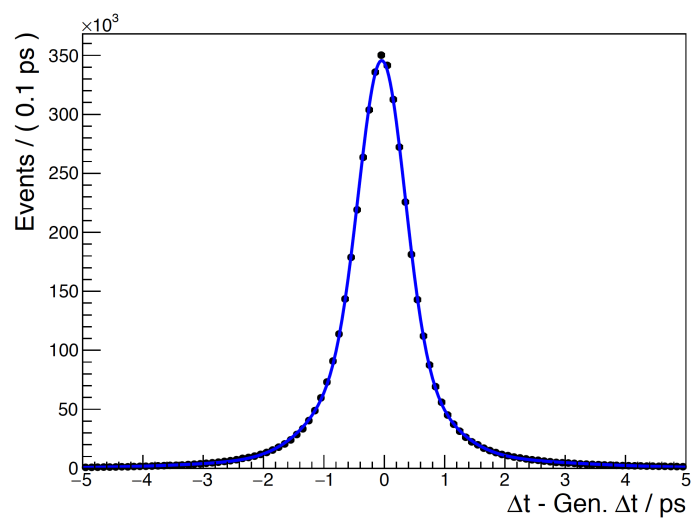

Figure 2: $\Delta t$ residuals for signal $B \rightarrow J / \psi K_{S}^{0}$ decays, with $J / \psi \rightarrow \mu^{+} \mu^{-}$and $K_{S}^{0} \rightarrow \pi^{+} \pi^{-}[1]$.

\section{Prospects for the angle $\phi_{1}$}

The $\phi_{1}$ angle of the unitarity triangle is defined by CKM matrix elements as being $\phi_{1}=$ $\arg \left[-V_{c b}^{*} V_{c d} /\left(V_{t b}^{*} V_{t d)}\right]\right.$. It is the most precisely measured $C P$ violating quantity and as such it is a very important input in the global CKM fit to test the standard model. The most precise measurement is achieved with the $B^{0} \rightarrow J / \psi K_{S}^{0}$ decay, thanks to its relatively large branching ratio and its clear signature with four tracks, and also because it is dominated by a tree diagram. Currently the $C P$ parameter $\mathscr{S}_{C P}$ is still statistically limited but already in a near future with $5 \mathrm{ab}^{-1}$ of Belle II data, the systematic uncertainty due to the penguin contribution will become annoying. However it will be possible to control it with a good accuracy thanks to improved time-dependent analysis of $B^{0} \rightarrow J / \psi \pi^{0}$ decays. The final uncertainty reached on $\phi_{1}$ is expected to be better than $0.1^{\circ}$ with the full Belle II dataset, to be compared to the current world average precision of $\phi_{1}^{\text {w.a. }}=(22.2 \pm 0.7)^{\circ}$ [2], and to the global fit $\phi_{1}^{f i t}=\left(22.51_{-0.40}^{+0.55}\right)^{\circ}$ [3]. 
As far as charmless decays are concerned, they will not help improving the precision on $\phi_{1}$, since they occur through penguin diagrams featuring possible different CKM phases and resulting in an additional uncertainty. Their interest stays rather in their sensitivity to new physics contributions. Many progresses will be made possible in Belle II, and in particular all $K_{S}^{0}$ and $\phi$ decays will be used, like $\phi \rightarrow \pi^{+} \pi^{-} \pi^{0}$ that was never used before. The $\phi$ vertex reconstruction, which is made difficult in particular in the $K^{+} K^{-}$decay mode because of the small angle between the two kaons, will be improved using the beam-spot constraint. Many $B$ decay modes will be considered in Belle II, in particular $\phi K_{S}^{0}, \eta^{\prime} K_{S}^{0}, \omega K_{S}^{0}, K_{S}^{0} \pi^{0}$ and $K_{S}^{0} \pi^{0} \gamma$. There will be less or no competition from LHCb for many of these modes, and they will generally remain statistically limited even with the full Belle II dataset. Only for the $B^{0} \rightarrow \eta^{\prime} K_{S}^{0}$ decay, the statistical uncertainty will reach the systematic level with $50 \mathrm{ab}^{-1}$ of data. The new physics discovery potential of this penguin mode is illustrated with figure 3 .

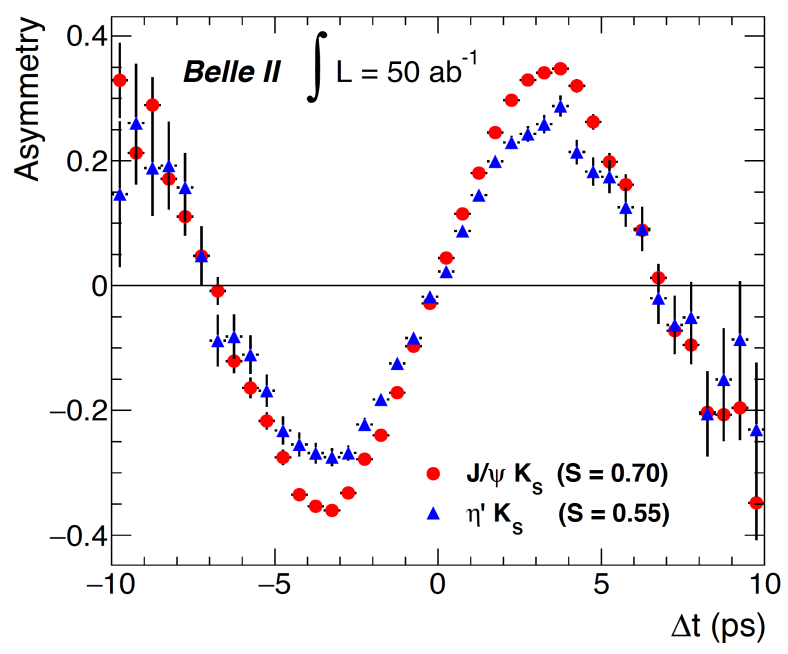

Figure 3: Time-dependent $C P$ asymmetries of $B^{0} \rightarrow J / \psi K_{S}^{0}$ (red dots) and $B^{0} \rightarrow \eta^{\prime} K_{S}^{0}$ decays (blue triangles) measured with a simulated full Belle II dataset including new physics [1].

\section{Prospects for the angle $\phi_{2}$}

The $\phi_{2}$ angle is defined as $\phi_{2}=\arg \left[-V_{t b}^{*} V_{t d} /\left(V_{u b}^{*} V_{u d)}\right]\right.$. It is measured through time-dependent $C P$ asymmetries of $B$ decays to $C P$ eigenstates governed by $b \rightarrow u$ transitions, that means $B$ decaying to light mesons. However in this case penguin diagrams contribute significantly, and direct $C P$ violation impact is not negligible anymore, leading to a shift of the measured $\phi_{2}$ angle. This shift can be estimated with an isospin analysis of $B \rightarrow \pi \pi$ and $B \rightarrow \rho \rho$ decays, based on Gronau-London relations among the different decay amplitudes [4].

With Belle II data it will be possible to reduce the ambiguity in the determination of $\phi_{2}$ (from an 8 -fold to a 4-fold or 2-fold ambiguity, depending on the central value), by measuring for the first time $B^{0} \rightarrow \pi^{0} \pi^{0}$ decays, though it is a very challenging analysis since the $B$ decay vertex must be reconstructed here with only two $\pi^{0}$. This will be possible in Belle II thanks to beam constraints and using $\gamma$ conversions and Dalitz $\pi_{D a l}^{0} \rightarrow \gamma e^{+} e^{-}$decays. Figure 4 shows the expected $\Delta t$ residual 
measured with simulated $B^{0} \rightarrow \pi^{0} \pi^{0}$ signal implying Dalitz $\pi_{D a l}^{0}$ decays. It is only about $50 \%$ worse than what is achieved with charged particles in the final state, as it was shown with figure 2.

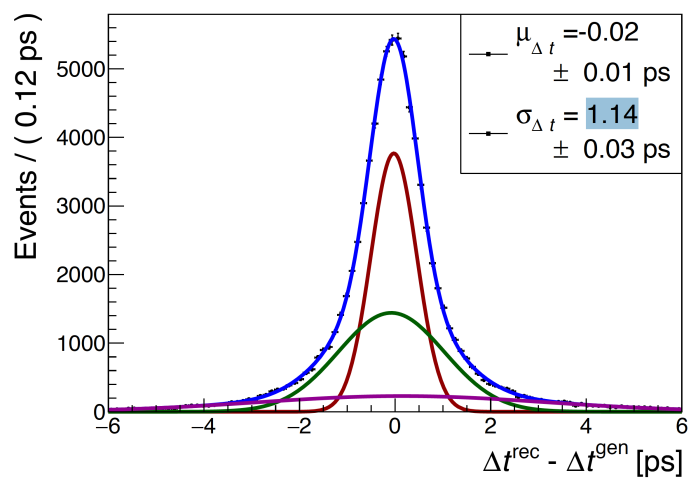

Figure 4: $\Delta t$ residuals of reconstructed signal $B^{0} \rightarrow \pi^{0} \pi^{0}$ decays with reconstructed Dalitz $\pi_{\text {Dal }}^{0}$ decays in Belle II simulated Dalitz $\pi_{D a l}^{0}$ events [1].

The final precision expected on $\phi_{2}$ with the full Belle II dataset will be better than $1^{\circ}$, that can be compared to the current world average precision of $\phi_{2}^{\text {w.a. }}=\left(84.9_{-4.5}^{+5.1}\right)^{\circ}$ [2], and to the global fit $\phi_{2}^{f i t}=\left(91.6_{-1.1}^{+1.7}\right)^{\circ}[3]$.

\section{Prospects for the angle $\phi_{3}$}

The $\phi_{3}$ angle is defined as $\phi_{3}=\arg \left[-V_{u b}^{*} V_{u d} /\left(V_{c b}^{*} V_{c d)}\right]\right.$. It can be determined with $B^{ \pm} \rightarrow D K^{ \pm}$ decays, implying interfering pure tree-mediated $b \rightarrow u$ and $b \rightarrow c$ transitions, and hence allowing a very clean theoretical prediction. However on the experimental side there is a lot of room to improve its accuracy: the current world average of $\phi_{3}^{\text {w.a. }}=\left(71.1_{-5.3}^{+4.6}\right)^{\circ}[2]$ still makes possible new physics contributing at the level of about $5^{\circ}$, motivating the targeted $1^{\circ}$ precision by combining Belle II and LHCb full datasets.

The considered decay modes have small branching fractions of the order of $0.01 \%$, and as many as possible $D$ decays must be considered. Thanks to an improved $K / \pi$ separation and a better continuum suppression in Belle II, in addition to a good capability to reconstruct neutrals, many yet-unused $D^{*}$ decay modes will be also considered with increasing statistics, like $D^{0} \pi^{0}, D^{0} \gamma$, $\pi^{0} \pi^{0}, K_{L}^{0} \pi^{0}, K_{S}^{0} \pi^{0} \pi^{0}, K_{S}^{0} K_{S}^{0} K_{L}^{0}$

The Belle II most sensitive analysis uses the self-conjugate $D$ decay to the 3-body final state $D \rightarrow K_{s}^{0} \pi^{+} \pi^{-}$, upon a method called the GGSZ method [5]. Other methods called the ADS method [6] and the GLW method [7] will be used to improve the global precision on $\phi_{3}$, based on different final states. Each method leads to particular ambiguities and different sensitivities, and the ultimate precision will be achieved by combining all methods and Belle II and LHCb measurements. The GGSZ analysis is a model-independent binned Dalitz plot analysis. It uses information on phase differences between symmetric Dalitz bins as an external input. This input is needed from future BES-III measurements based on $10 \mathrm{fb}^{-1}$ of $e^{+} e^{-}$collisions at the $D \bar{D}$ threshold, because currently available CLEO- $c$ measurements do not match the precision targeted by Belle II. One major source of systematic uncertainty impacting the $\phi_{3}$ measurement by Belle was due to the amplitude model used in the Dalitz analysis. In Belle II it will be avoided by the binning of the Dalitz plot, made 


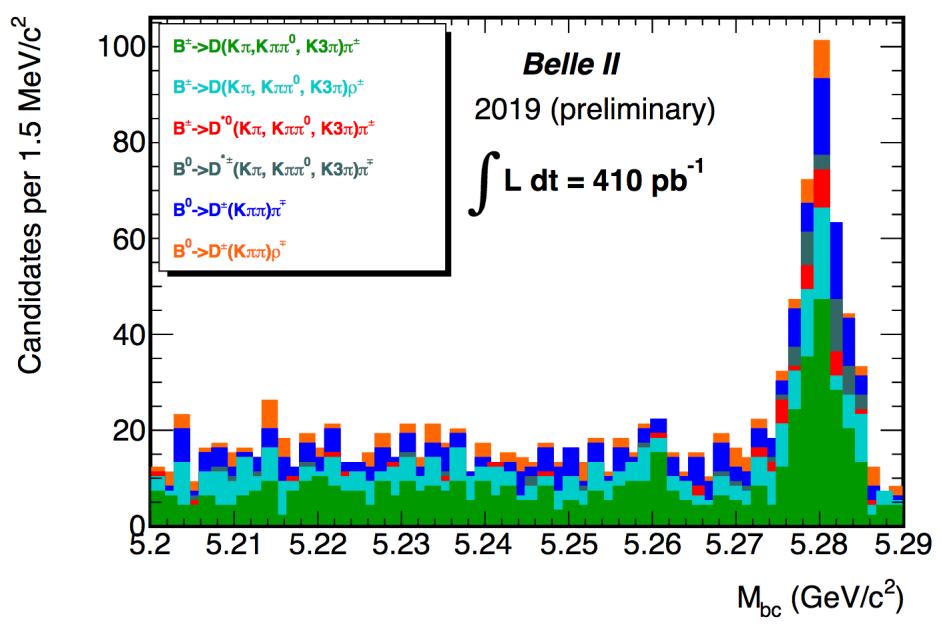

Figure 5: Beam-constrained $M_{b c}$ distribution of $B \rightarrow D^{(*)} h$ candidates with $h=\pi, \rho$, reconstructed with first $0.41 \mathrm{fb}^{-1}$ of Belle II data registered in 2019.

possible thanks to a larger bin population. Systematics level will be further improved thanks to $B \rightarrow D \pi$ decays, that will be abundantly reconstructed. They are not very sensitive to $\phi_{3}$ but will allow to control shape models used in the analysis. Figure 5 shows first $B \rightarrow D^{(*)} h$ decays reconstructed with early Belle II data in 2019, where $h=\pi$ or $\rho$. Finally, a key asset of Belle II for such analyses is its unbiased trigger.

By 2027, an uncertainty on $\phi_{3}$ at the level of $3^{\circ}$ is expected with this golden mode, and conservatively well below $2^{\circ}$ after combination with all Belle II measurements based on other $B$ decay modes, with still room for improvement. This prospect can be compared to the current precision of the Belle combined $\phi_{3}$ result, which is $13^{\circ}$.

\section{Conclusion and outlook}

Belle II has successfully started physics in 2019 and there are opportunities for important results already on the short term. In particular the three CKM $b d$ unitarity triangle angle uncertainties are expected to be improved significantly already in the coming years. In the end of Belle II with a full dataset corresponding to an integrated luminosity of $50 \mathrm{ab}^{-1}$, many measurements will start to be systematically limited, and lots of work will be necessary as well on the experimental side and on the theoretical one to meet the challenge. This huge dataset comes along with an important pollution by machine induced background, and a huge effort is made to mitigate its impact on the Belle II physics performances.

\section{References}

[1] The Belle-II Collaboration (E. Kou, et al.), "The Belle II Physics Book", arXiv:1808.10567 [hep-ex], submitted to PTEP.

[2] Heavy Flavour Averaging Group, https://hflav.web.cern.ch/

[3] CKMfitter group, http://ckmfitter.in2p3.fr/ 
[4] M. Gronau and D. London, "Isospin analysis of $C P$ asymmetries in B decays", Phys. Rev. Lett. 65 (1990) 3381-3384.

[5] A. Giri, Y. Grossman, A. Soffer and J. Zupan, "Determining gamma using $B^{ \pm} \rightarrow D K^{ \pm}$with multibody D decays", Phys. Rev. D 68 (2003) 054018.

[6] D. Atwood, I. Dunietz and A. Soni, "Enhanced $C P$ violation with $B \rightarrow K D^{0}\left(\bar{D}^{0}\right)$ modes and extraction of the CKM angle $\gamma^{\prime \prime}$, Phys. Rev. Lett. 78 (1997) 3257-3260.

[7] M. Gronau and D. Wyler, "On determining a weak phase from $C P$ asymmetries in charged $B$ decays", Phys. Lett. B265, 172 (1991);

M. Gronau and D. London, "How to determine all the angles of the unitarity triangle from $B_{(d)}^{0} \rightarrow D K_{(S)}$ and $B_{(s)}^{0} \rightarrow D^{0 ", ~ P h y s . ~ L e t t . ~ B 253, ~} 483$ (1991). 